PROCEEDINGS OF THE

AMERICAN MATHEMATICAL SOCIETY

Volume 131, Number 8, Pages 2553-2560

S 0002-9939(03)07229-0

Article electronically published on March 17, 2003

\title{
ON THE BARTLE-GRAVES THEOREM
}

\author{
J. M. BORWEIN AND A. L. DONTCHEV \\ (Communicated by N. Tomczak-Jaegermann) \\ Dedicated to Bob Bartle
}

\begin{abstract}
The Bartle-Graves theorem extends the Banach open mapping principle to a family of linear and bounded mappings, thus showing that surjectivity of each member of the family is equivalent to the openness of the whole family. In this paper we place this theorem in the perspective of recent concepts and results, and present a general Bartle-Graves theorem for set-valued mappings. As applications, we obtain versions of this theorem for mappings defined by systems of inequalities, and for monotone variational inequalities.
\end{abstract}

Half a century ago R. G. Bartle and L. M. Graves published in [1] a generalization of the Banach open mapping principle of the following (slightly updated) form. Let $X$ and $Y$ be real Banach spaces, let $T$ be a compact metric space, let $\mathcal{L}(X, Y)$ be the space of linear and bounded mappings from $X$ to $Y$, and let $C(T, X)$ be the space of continuous functions from $T$ to $X$ endowed with the supremum norm.

Theorem 1 ([1], Theorem 4, [6], p. 85). Let $A: T \rightarrow \mathcal{L}(X, Y)$ be a continuous operator-valued function. Define the mapping $\mathbf{A}: C(T, X) \rightarrow C(T, Y)$ by

$$
(\mathbf{A} x(\cdot))(t):=A(t) x(t) \text { for } t \in T .
$$

Then $\mathbf{A} \in \mathcal{L}(C(T, X), C(T, Y))$ and the following are equivalent:

(i) For every $t \in T$ the mapping $A(t)$ is surjective.

(ii) There exists a constant $N>0$ such that for every $y(\cdot) \in C(T, Y)$ there exists $x(\cdot) \in C(T, X)$ with $\mathbf{A} x(\cdot)=y(\cdot)$ and $\|x(t)\| \leq N\|y(t)\|$ for all $t \in T$.

The Banach open mapping principle is obtained from this theorem by considering that $T$ consists of only one element.

In a different direction, the Banach open mapping principle found its extension for nonlinear operators in the work of Graves [7] and, merging with a related earlier result by Lusternik [9], in the 60s and 70s it became a cornerstone of the theory of minimum problems with constraints. The underlying concept was then recognized as the property of metric regularity which is formulated in the more abstract setting of set-valued mappings.

In this paper $\|\cdot\|$ is any norm and $\mathbb{B}$ is a closed unit ball; a ball centered at $a$ with radius $r$ is $\mathbb{B}_{r}(a)$. The distance from a point $x$ to a set $A$ is denoted by $d(x, A)$. We indicate by $F: X \rightrightarrows Y$ a set-valued mapping from $X$ to the subsets of $Y$. If $F$ is a function, that is, for each $x \in X$ the set of values $F(x)$ consists of

Received by the editors June 11, 2002 and, in revised form, February 12, 2003.

2000 Mathematics Subject Classification. Primary 49J53, 46N10, 47H04, 54C60. 
no more than one element, then we write $F: X \rightarrow Y$. The graph of a mapping $F$ is given by $\operatorname{gph} F=\{(x, y) \mid y \in F(x)\}$ and its inverse $F^{-1}$ is defined as $x \in F^{-1}(y) \Leftrightarrow y \in F(x)$.

A set-valued mapping $F: X \rightrightarrows Y$ with $(\bar{x}, \bar{y}) \in \operatorname{gph} F$ is said to be metrically regular at $\bar{x}$ for $\bar{y}$ if there exists a constant $\kappa>0$ such that

$$
d\left(x, F^{-1}(y)\right) \leq \kappa d(y, F(x)) \text { for all }(x, y) \text { close to }(\bar{x}, \bar{y}) .
$$

The infimum of $\kappa$ for which (1) holds is the modulus of metric regularity which we denote by reg $F(\bar{x} \mid \bar{y})$; the case when $F$ is not metrically regular corresponds to $\operatorname{reg} F(\bar{x} \mid \bar{y})=\infty$. If $A: X \rightarrow Y$ is linear and bounded, then metric regularity of $A$ at any point in its graph is equivalent to metric regularity at zero for zero; for simplicity we then denote the modulus of metric regularity of $A$ by $\operatorname{reg} A$. This phenomenon, and an adjoint duality formula, extends to closed convex processes 3]. Metric regularity of a mapping $F$ at $\bar{x}$ for $\bar{y}$ with a constant $\kappa$ is known to be equivalent to the so-called Aubin property of the inverse $F^{-1}$ at $\bar{y}$ for $\bar{x}$ with the same constant $\kappa$. That is, equivalently, there exists a neighborhood $O$ of $\bar{x}$ such that

$$
F^{-1}\left(y^{\prime}\right) \cap O \subset F^{-1}(y)+\kappa\left\|y^{\prime}-y\right\| \mathbb{B} \quad \text { for all } y^{\prime}, y \text { close to } \bar{y} .
$$

In the sequel we shall also use the fact that the metric regularity of $F$ at $\bar{x}$ for $\bar{y}$ implies that for a sufficiently small neighborhood $O$ of $\bar{x}, F^{-1}(y) \cap O \neq \emptyset$ for all $y$ close to $\bar{y}$. For recent works on metric regularity, see [4], [5], [8] and [11].

Recall that $g: X \rightarrow Y$ is strictly differentiable at $\bar{x} \in \operatorname{dom} g$ with a strict derivative $D g(\bar{x}) \in \mathcal{L}(X, Y)$ if for every $\varepsilon>0$ there exists $\delta>0$ such that

$$
\left\|g\left(x_{1}\right)-g\left(x_{2}\right)-D g(\bar{x})\left(x_{1}-x_{2}\right)\right\|<\varepsilon\left\|x_{1}-x_{2}\right\| \text { whenever } x_{1}, x_{2} \in \mathbb{B}_{\delta}(\bar{x}) .
$$

An updated form of the results of Lusternik and Graves is as follows:

Theorem 2 (Lusternik-Graves). Let $g: X \rightarrow Y$ be strictly differentiable at $\bar{x}$ with strict derivative $D g(\bar{x})$, and let $G: X \rightrightarrows Y$. Let $(\bar{x}, \bar{y}) \in \operatorname{gph}(g+G)$, and let $\operatorname{gph} G$ be closed in a neighborhood of $(\bar{x}, \bar{y})$. Then

$$
\operatorname{reg}(g+G)(\bar{x} \mid \bar{y})=\operatorname{reg}(D g+G)(\bar{x} \mid \bar{y}) .
$$

In particular, $g$ is metrically regular at $\bar{x}$ for $g(\bar{x})$ if and only if $D g(\bar{x})$ is surjective.

In terms of the modulus of metric regularity, the Bartle-Graves theorem can be formulated in the following concise form (which does not follow directly from Theorem 1 but is contained in the original proof of Bartle and Graves):

Theorem 1a (Bartle-Graves reformulated). In the notation of Theorem 1,

$$
\operatorname{reg} \mathbf{A}=\sup _{t \in T} \operatorname{reg} A(t) .
$$

Here and below, it is instructive to consider the case where $T$ is either (i) the onepoint (Alexandroff) compactification, $\overline{\mathbf{N}}$, or (ii) the Céch compactification, $\beta(\mathbf{N})$, of $\mathbf{N}$. In the latter case recall that $C(\beta(\mathbf{N}))$ is isometric to $\ell^{\infty}(\mathbf{N})$, so that the continuity hypothesis becomes one of boundedness.

A new proof of the Bartle-Graves theorem was recently given by Páles [10] based on the Michael selection theorem. In this note we extend Páles' proof to provide a more general version of the theorem for set-valued mappings. We then apply it to systems of inequalities and to monotone variational inequalities. 
Consider a continuous function $f: T \times X \rightarrow Y$, and let $\bar{x} \in C(T, X)$. For a fixed $t \in T$, let reg $f(t ; \bar{x}(t))$ denote the modulus of metric regularity of $f(t, \cdot)$ at $\bar{x}(t)$ for $f(t, \bar{x}(t))$. Define $\mathbf{f}: C(T, X) \rightarrow C(T, Y)$ as

$$
(\mathbf{f}(x(\cdot)))(t)=f(t, x(t)) \text { for } t \in T,
$$

and denote the modulus of metric regularity of $\mathbf{f}$ at $\bar{x}(\cdot)$ for $\mathbf{f}(\bar{x}(\cdot))$ by $\operatorname{reg} \mathbf{f}(\bar{x}(\cdot))$. Recall that $\mathcal{T}$ is an open tube around $\bar{x}(\cdot)$ if $\mathcal{T}=\{(t, x) \in T \times X \mid\|x-\bar{x}(t)\|<\delta\}$ for some $\delta>0$. The next lemma gives a sufficient condition for strict differentiability of the induced function, $\mathbf{f}$.

Lemma 3. Let $\bar{x}(\cdot) \in C(T, X)$, and let $f: T \times X \rightarrow Y$ be continuous with respect to $t$ and Fréchet differentiable with respect to $x$ in an open tube $\mathcal{T}$ around $\bar{x}(\cdot)$ such that the derivative $D_{x} f$ is continuous in $\mathcal{T}$. Then the function $\mathbf{f}$ is strictly differentiable at $\bar{x}(\cdot)$, in the norms of $C(T, X)$ and $C(T, Y)$, with strict derivative

$$
(D \mathbf{f}(\bar{x}(\cdot)))(t)=D_{x} f(t, \bar{x}(t)) \text { for } t \in T .
$$

Proof. Apply the definition of the strict differentiability (2) to $\mathbf{f}$.

We are now ready for the following result which is an extension of the BartleGraves theorem for smooth functions:

Theorem 4. Under the conditions of Lemma 3,

$$
\operatorname{reg} \mathbf{f}(\bar{x}(\cdot))=\sup _{t \in T} \operatorname{reg} f(t ; \bar{x}(t))=\sup _{t \in T} \operatorname{reg} D_{x} f(t, \bar{x}(t))=\operatorname{reg} D \mathbf{f}(\bar{x}(\cdot)) .
$$

Proof. From the Lusternik-Graves theorem (Theorem 2) we obtain that

$$
\operatorname{reg} \mathbf{f}(\bar{x}(\cdot))=\operatorname{reg} D \mathbf{f}(\bar{x}(\cdot)) \text { and } \operatorname{reg} f(t ; \bar{x}(t))=\operatorname{reg} D_{x} f(t, \bar{x}(t)) .
$$

Combining these equalities with Lemma 3 and the Bartle-Graves theorem (Theorem 1a) completes the proof.

Let $F: T \times X \rightrightarrows Y$ be a set-valued mapping. Define, much as before, a set-valued mapping $\mathbf{F}$ acting from $C(T, X)$ into the subsets of $C(T, Y)$ as follows:

$$
\mathbf{F}(x(\cdot))=\{y(\cdot) \in C(T, Y) \mid y(t) \in F(t, x(t)) \text { for all } t \in T\} .
$$

The main result of this paper will be given in Theorem 6 . A first step towards it is the next proposition.

Proposition 5. If $\bar{x}(\cdot) \in C(T, X), \bar{y}(\cdot) \in C(T, Y)$ and $(\bar{x}(t), \bar{y}(t)) \in \operatorname{gph} F(t, \cdot)$ for $t \in T$, then

$$
\operatorname{reg} \mathbf{F}(\bar{x}(\cdot) \mid \bar{y}(\cdot)) \geq \sup _{t \in T} \operatorname{reg} F(t ; \bar{x}(t) \mid \bar{y}(t)) .
$$

Proof. The case $\sup _{t \in T} \operatorname{reg} F(t ; \bar{x}(t) \mid \bar{y}(t))=0$ is trivial. Take

$$
\gamma<\sup _{t \in T} \operatorname{reg} F(t ; \bar{x}(t) \mid \bar{y}(t))
$$

then for some $t_{0} \in T, \gamma<\operatorname{reg} F\left(t_{0} ; \bar{x}\left(t_{0}\right) \mid \bar{y}\left(t_{0}\right)\right)$. This implies that for any neighborhoods $V$ of $\bar{y}\left(t_{0}\right)$ and $U$ of $\bar{x}\left(t_{0}\right)$ there exist $y_{1}, y_{2} \in V, y_{1} \neq y_{2}$, and $x_{1} \in U$ with $y_{1} \in F\left(t_{0}, x_{1}\right)$ such that for every $x_{2}$ with $y_{2} \in F\left(t_{0}, x_{2}\right)$ we have

$$
\frac{\left\|x_{2}-x_{1}\right\|}{\left\|y_{1}-y_{2}\right\|}>\gamma .
$$


Fix neighborhoods $U$ and $V$ and associated points $y_{1}, y_{2}, x_{1}$. Take neighborhoods $\mathcal{U} \subset C(T, X)$ and $\mathcal{V} \subset C(T, Y)$ of $\bar{x}(\cdot)$ and $\bar{y}(\cdot)$, respectively, of the "sizes" of $U$ and $V$; that is, if $\mathbb{B}_{a}\left(\bar{x}\left(t_{0}\right)\right) \subset U$, then $\mathbb{B}_{a}(\bar{x}(\cdot)) \subset \mathcal{U}$, and similarly for $\mathcal{V}$.

Next, choose functions $y_{1}(\cdot), y_{2}(\cdot) \in \mathcal{V}$ with

$$
\left\|y_{1}(\cdot)-y_{2}(\cdot)\right\|=\max _{t \in T}\left\|y_{1}(t)-y_{2}(t)\right\|=\left\|y_{1}\left(t_{0}\right)-y_{2}\left(t_{0}\right)\right\|=\left\|y_{1}-y_{2}\right\| .
$$

Let $x_{1}(\cdot)$ be a function in $\mathcal{V}$ with $y_{1}(t) \in F\left(t, x_{1}(t)\right), t \in T$, and such that $x_{1}\left(t_{0}\right)=$ $x_{1}$. [If such a function $x_{1}(\cdot)$ does not exist, then, since the neighborhoods $\mathcal{U}$ and $\mathcal{V}$ can be chosen arbitrarily small, we conclude that $\operatorname{reg} \mathbf{F}(\bar{x}(\cdot) \mid \bar{y}(\cdot))=\infty$ and there is nothing more to prove.] From (5) and (6), for any function $x_{2}(\cdot)$ with $y_{2}(\cdot) \in \mathbf{F}\left(x_{2}(\cdot)\right)$, we have

$$
\begin{aligned}
\left\|x_{2}(\cdot)-x_{1}(\cdot)\right\| & \geq\left\|x_{2}\left(t_{0}\right)-x_{1}\left(t_{0}\right)\right\|=\left\|x_{2}-x_{1}\right\| \\
& \geq \gamma\left\|y_{2}-y_{1}\right\|=\gamma\left\|y_{1}\left(t_{0}\right)-y_{2}\left(t_{0}\right)\right\|=\gamma\left\|y_{1}(\cdot)-y_{2}(\cdot)\right\| .
\end{aligned}
$$

Hence, $\operatorname{reg} \mathbf{F}(\bar{x} \mid \bar{y}) \geq \gamma$ and therefore (4) holds.

Recall that a mapping $G: T \rightrightarrows T$ is (sequentially) lower semicontinuous on $T$ if for every $t \in T$, every $x \in G(t)$ and every sequence $t_{k} \in T, t_{k} \rightarrow t$, there exist $x_{k} \in G\left(t_{k}\right)$ for $k=1,2, \ldots$, with $x_{k} \rightarrow x$. In our setting, sequential lower semicontinuity and lower semicontinuity coincide.

Theorem 6. The inequality (4) becomes an equality if there exists a constant a $>0$ such that the mapping $P$ defined as

$$
T \times Y \ni(t, y) \mapsto P(t, y):=F(\cdot, t)^{-1}(y) \cap \mathbb{B}_{a}(\bar{x}(t))
$$

satisfies the following two conditions:

a) For every $t \in T$ and every $y \in \mathbb{B}_{a}(\bar{y}(t)), P(t, y)$ is a closed, and convex set.

b) For every $y \in \bigcup_{t \in T} \mathbb{B}_{a}(\bar{y}(t))$ the mapping $T \ni t \mapsto P(t, y)$ is lower semicontinuous on $T$.

Proof. If $\sup _{t \in T} \operatorname{reg} F(t ; \bar{x}(t) \mid \bar{y}(t))=\infty$, then equality follows from (4) and there is nothing to prove. Let $\gamma$ and $\beta$ be positive numbers such that $\gamma>\gamma-\beta>$ $\sup _{t \in T} \operatorname{reg} F(t ; \bar{x}(t) \mid \bar{y}(t))$. Then for every $t \in T$ the mapping $F(t, \cdot)$ is metrically regular with constant $\gamma-\beta$ which is independent of $t$. Since metric regularity is equivalent to the Aubin property with the same constant, for every $t \in T$ there exist neighborhoods $U(t)$ and $V(t)$ of $\bar{x}(t)$ and $\bar{y}(t)$ respectively, such that for every $y, y^{\prime} \in V(t)$ and $x \in U(t)$ with $y \in F(t, x)$, there exists $x^{\prime}$ with $y^{\prime} \in F\left(t, x^{\prime}\right)$ such that $\left\|x^{\prime}-x\right\| \leq(\gamma-\beta)\left\|y^{\prime}-y\right\|$.

Compactness of $T$ allows us to choose neighborhoods $\mathcal{U}, \mathcal{V}$ of $\bar{x}(\cdot)$ and $\bar{y}(\cdot)$ in $C(T, X)$ and $C(T, Y)$ respectively, such that if $x(\cdot) \in \mathcal{U}$, then $x(t) \in U(t)$ for every $t \in T$ and also for $V$ and $\mathcal{V}$. Take any functions $y(\cdot), y_{1}(\cdot) \in \mathcal{V}$ and $x_{1}(\cdot) \in \mathcal{U}$ such that $y_{1}(t) \in F\left(x_{1}(t), t\right)$ for all $t \in T$. Consider the mapping

$$
T \ni t \mapsto M(t):=\left\{x \in X \mid F(x, t) \ni y(t) \text { and }\left\|x-x_{1}(t)\right\| \leq \gamma\left\|y(t)-y_{1}(t)\right\|\right\} .
$$

We shall now show that this mapping has nonempty, convex and closed values and is lower semicontinuous on $T$. Then, by the Michael selection theorem, there will exist a continuous selection $x(\cdot)$ of $M$. This, by the definition of $M$, means precisely that the mapping $\mathbf{F}$ has the Aubin property at $\bar{y}(\cdot)$ for $\bar{x}(\cdot)$ with constant $\gamma$. Therefore $\gamma \geq \operatorname{reg} \mathbf{F}(\bar{y}(\cdot) \mid \bar{x}(\cdot))$ and since $\gamma$ can be made arbitrarily close to 
$\sup _{t \in T} \operatorname{reg} F(t ; \bar{x}(t) \mid \bar{y}(t))$ we obtain the opposite inequality to (4), and hence the equality is established.

We proceed with proving that the mapping $t \mapsto M(t)$ has the desired properties. That the mapping $M$ has closed and convex values follows from condition a). Also, for all $t \in T$ the mapping $F(t, \cdot)$ is metrically regular at $\bar{x}(t)$ for $\bar{y}(t)$; hence $M(t) \neq \emptyset$ for all $t \in T$. It remains to show lower semicontinuity. Let $t \in T$ and $T \ni t_{k} \rightarrow t$, and let $x \in M(t)$. If $y(t)=y_{1}(t)$, we have $M(t)=x_{1}(t)$ and then $M\left(t_{k}\right) \ni x_{1}\left(t_{k}\right) \rightarrow$ $x_{1}(t)$, and we are done. Assume that for some $\alpha>0$ we have $\alpha<\left\|y(t)-y_{1}(t)\right\|$; then, without loss of generality, $\alpha / 2<\left\|y\left(t_{k}\right)-y_{1}\left(t_{k}\right)\right\|$ for all $k$. From condition b) there exists a sequence $\hat{x}_{k} \rightarrow x$ such that $y(t) \in F\left(t_{k}, \hat{x}_{k}\right)$ for all $k$. From the Aubin property of $F^{-1}\left(t_{k}, \cdot\right)$ at $\bar{y}\left(t_{k}\right)$ for $\bar{x}\left(t_{k}\right)$ there exists $\tilde{x}_{k} \in F^{-1}\left(t_{k}, \cdot\right)\left(y\left(t_{k}\right)\right)$ such that

$$
\left\|\tilde{x}_{k}-\hat{x}_{k}\right\| \leq \gamma\left\|y\left(t_{k}\right)-y(t)\right\| \text {. }
$$

Thus, $\tilde{x}_{k} \rightarrow x$ as $k \rightarrow \infty$. Again from the Aubin property of $F^{-1}\left(t_{k}, \cdot\right)$ with a constant $\gamma-\beta$, for each $k$ there exists $\check{x}_{k} \in F^{-1}\left(t_{k}, \cdot\right)\left(y\left(t_{k}\right)\right)$ such that

$$
\left\|\check{x}_{k}-x_{1}\left(t_{k}\right)\right\| \leq(\gamma-\beta)\left\|y\left(t_{k}\right)-y_{1}\left(t_{k}\right)\right\| .
$$

Let

$$
\varepsilon_{k}:=\frac{\left\|\hat{x}_{k}-x\right\|+\left\|x_{1}\left(t_{k}\right)-x_{1}(t)\right\|+2 \gamma\left\|y\left(t_{k}\right)-y(t)\right\|+\gamma\left\|y_{1}\left(t_{k}\right)-y_{1}(t)\right\|}{\left\|y\left(t_{k}\right)-y_{1}\left(t_{k}\right)\right\|} .
$$

Since $\hat{x}_{k} \rightarrow x$ and $x_{1}(\cdot), y(\cdot), y_{1}(\cdot)$ are continuous functions, and the denominator is $>\alpha / 2$, we have $\varepsilon_{k} \rightarrow 0$ as $k \rightarrow \infty$. Let $\alpha_{k}$ be a sequence of positive scalars convergent to zero which satisfy

$$
\alpha_{k} \geq \frac{\varepsilon_{k}}{\beta+\varepsilon_{k}}
$$

and consider the sequence

$$
x_{k}=\alpha_{k} \check{x}_{k}+\left(1-\alpha_{k}\right) \tilde{x}_{k} .
$$

Clearly, $x_{k} \rightarrow x$ as $k \rightarrow \infty$. Moreover, $y\left(t_{k}\right) \in F\left(t_{k}, x_{k}\right)$ because of the convexity assumption in a). Furthermore,

$$
\begin{aligned}
&\left\|x_{k}-x_{1}\left(t_{k}\right)\right\| \leq \alpha_{k}\left\|\check{x}_{k}-x_{1}\left(t_{k}\right)\right\|+\left(1-\alpha_{k}\right)\left\|\tilde{x}_{k}-x_{1}\left(t_{k}\right)\right\| \\
& \leq \alpha_{k}(\gamma-\beta)\left\|y\left(t_{k}\right)-y_{1}\left(t_{k}\right)\right\|+\left(1-\alpha_{k}\right)\left(\left\|\tilde{x}_{k}-\hat{x}_{k}\right\|+\left\|\hat{x}_{k}-x\right\|\right. \\
&\left.+\left\|x-x_{1}(t)\right\|+\left\|x_{1}(t)-x_{1}\left(t_{k}\right)\right\|\right) \\
& \leq \alpha_{k}(\gamma-\beta)\left\|y\left(t_{k}\right)-y_{1}\left(t_{k}\right)\right\|+\left(1-\alpha_{k}\right)\left(\gamma\left\|y\left(t_{k}\right)-y(t)\right\|+\left\|\hat{x}_{k}-x\right\|\right. \\
&\left.+\gamma\left\|y(t)-y_{1}(t)\right\|+\left\|x_{1}(t)-x_{1}\left(t_{k}\right)\right\|\right) \\
& \leq \alpha_{k}(\gamma-\beta)\left\|y\left(t_{k}\right)-y_{1}\left(t_{k}\right)\right\|+\left(1-\alpha_{k}\right)\left(\gamma\left\|y\left(t_{k}\right)-y(t)\right\|+\left\|\hat{x}_{k}-x\right\|\right. \\
&\left.+\gamma\left\|y\left(t_{k}\right)-y_{1}\left(t_{k}\right)\right\|+\gamma\left\|y\left(t_{k}\right)-y(t)\right\|+\gamma\left\|y_{1}\left(t_{k}\right)-y_{1}(t)\right\|+\left\|x_{1}(t)-x_{1}\left(t_{k}\right)\right\|\right) \\
& \leq\left(\alpha_{k}(\gamma-\beta)+\left(1-\alpha_{k}\right)\left(\gamma+\varepsilon_{k}\right)\right)\left\|y\left(t_{k}\right)-y_{1}\left(t_{k}\right)\right\| .
\end{aligned}
$$

Because of the choice of $\alpha_{k}$ we have $\alpha_{k}(\gamma-\beta)+\left(1-\alpha_{k}\right)\left(\gamma+\varepsilon_{k}\right) \leq \gamma$, hence

$$
\left\|x_{k}-x_{1}\left(t_{k}\right)\right\| \leq \gamma\left\|y\left(t_{k}\right)-y_{1}\left(t_{k}\right)\right\|
$$

and therefore $x_{k} \in M\left(t_{k}\right)$. This completes the proof.

Conditions a) and b) are not necessary for the conclusion of the theorem; they are needed in the proof to apply the Michael selection theorem. On the other hand they are quite general, e.g., in the context of variational analysis. We illustrate 
this result with two applications where these conditions are automatically satisfied - and they hold in the original setting of Bartle and Graves.

Example 7. Systems of inequalities. As a first example of application, consider the mapping

$$
T \times X \ni(t, x) \mapsto F(t, x):=f(t, x)+K,
$$

where $f: T \times X \rightarrow \mathbb{R}^{m}$ and $K$ is a closed and convex cone in $\mathbb{R}^{m}$.

Inducing $\mathbf{F}(x(\cdot))$ on the basis of $F(t, x)$ as in $(3)$, we have $(\bar{x}(\cdot), \bar{y}(\cdot)) \in \operatorname{gph} \mathbf{F}$ when $\bar{x}(\cdot)$ and $\bar{y}(\cdot)$ are continuous functions which satisfy the inequality $f(t, \bar{x}(t)) \leq_{K}$ $\bar{y}(t)$ for all $t \in T$, where $\leq_{K}$ is the order relation defined by $K$. Metric regularity of mappings of the form (7) is a central issue in the analysis of optimization problems with constraints given by order inequalities; see [2] for a detailed earlier discussion of this topic.

For simplicity, we let $\bar{x}(\cdot)=0, \bar{y}(\cdot)=0$ and $f(t, 0)=0$ for all $t \in T$, and assume that

(*) For some neighborhood $O$ of the origin in $X$ the function $f$ :

$T \times X \rightarrow \mathbb{R}^{m}$ is continuous and Fréchet differentiable with respect

to $x$ in $T \times O$ and its derivative $D_{x} f$ is continuous there.

Then metric regularity of $\mathbf{F}$ at zero for zero is, according to Theorem 2, equivalent to metric regularity of the linearization of $\mathbf{F}$, that is, of the mapping $\mathbf{S}$ defined as in (3) on the basis of the "pointwise" mapping $S(t, x)=A(t) x+K$ with $A(t)=$ $D_{x} f(t, 0)$. The mapping $S: T \times X \rightrightarrows Y$ has closed graph and for each $t \in T$, $\operatorname{gph} S(t, \cdot)$ is a convex and closed cone in $X \times \mathbb{R}^{m}$. Hence condition a) in Theorem 6 holds for the mapping $S$.

Let $\sup _{t \in T} \operatorname{reg} S(t ; 0 \mid 0)<\infty$, otherwise, by Proposition 5 , there is nothing to prove. In terms of the Aubin property, there exists a constant $\kappa>0$ such that for every $t \in T, y, y^{\prime}$ close to the origin and $x$ with $A(t) x \leq y$, there exists an $x^{\prime}$ with $A(t) x^{\prime} \leq y^{\prime}$ such that $\left\|x^{\prime}-x\right\| \leq \kappa\left\|y^{\prime}-y\right\|$. Let $t_{k} \rightarrow t \in T$ and let $x \in S^{-1}(t, \cdot)(y)$ for some $(x, y)$ close to the origin. Now, clearly $A\left(t_{k}\right) x \leq y+\left(A\left(t_{k}\right)-A(t)\right) x$ for all $k$. From the Aubin continuity of $S^{-1}\left(t_{k}, \cdot\right)$ there exists a sequence $x_{k}$ with $A\left(t_{k}\right) x_{k} \leq y$ such that $\left\|x_{k}-x\right\| \leq \kappa\left\|\left(A\left(t_{k}\right)-A(t)\right) x\right\|$, and hence the continuity of $A$ implies that $x_{k} \rightarrow x$, that is, condition b) of Theorem 6 holds. Thus, we obtain the equality

$$
\operatorname{reg} \mathbf{F}(\bar{x}(\cdot) \mid \bar{y}(\cdot))=\operatorname{reg} \mathbf{S}(\bar{x}(\cdot) \mid \bar{y}(\cdot))=\sup _{t \in T} \operatorname{reg} S(t, \cdot)(\bar{x}(t) \mid \bar{y}(t)) .
$$

For $K=\{0\}$, from the second equality we recover the Bartle-Graves theorem (Theorem 1a).

Example 8. Monotone variational inequalities. Observe that both conditions a) and $\mathrm{b}$ ) in Theorem 6 are trivially satisfied if for $t \in T$ and $y$ near $\bar{y}$ the mapping $P$ is single-valued and the function $P(\cdot, y)$ is continuous. Such a property of $P$ automatically follows from the metric regularity of $F$ if $F$ is used to describe a monotone variational inequality.

Specifically, let $f: T \times X \rightarrow X^{*}$ be a continuous function, and let $Q$ be a convex and closed set in $X$. Consider the following variational inequality: find $x(\cdot) \in C(T, X)$ such that for every $t \in T$,

$$
\langle f(t, x(t)), v-x(t)\rangle \geq 0 \quad \text { for all } v \in Q .
$$


This variational inequality can be written as the inclusion

$$
0 \in F(t, x(t)):=f(t, x(t))+N_{Q}(x(t))
$$

where $N_{Q}(x)$ is the standard normal cone to $Q$ at $x$. Again, for simplicity assume that $F(t, 0) \ni 0$ for all $t \in T$; that is, our reference point is $\bar{x}(\cdot)=0, \bar{y}(\cdot)=0$. Also, assume that condition $(*)$ in Example 7 holds for the current $f$ and, moreover, that the derivative $A(t):=D_{x} f(t, 0)$ is negative semidefinite for all $t \in T$, that is, $\langle A(t) x, x\rangle \leq 0$ for all $x \in X$ and $t \in T$. Since metric regularity is stable under linearization, in the sense of Theorem 2, we may replace $F$ by the mapping $(t, x) \mapsto S(t, x):=A(t) x+N_{Q}(x)$ which is associated with the linearized variational inequality $\langle A(t) x(t), v-x(t)\rangle \geq 0$ for all $v \in Q$ and for each $t \in T, S(t, \cdot)$ is a monotone mapping. Define $\mathbf{F}$ as in (3) and, without loss of generality, assume that $\sup _{t \in T} \operatorname{reg} S(t ; \bar{x}(t) \mid \bar{y}(t))<\infty$. At this point we apply the well-known fact that any monotone mapping which is lower semicontinuous is single-valued. The application of this fact to metrically regular mappings has the form:

Let $H: X \rightrightarrows X^{*}$ be monotone locally around $(\bar{x}, \bar{y}) \in \operatorname{gph} H$, that is, $\left\langle y_{1}-y_{2}, x_{1}-x_{2}\right\rangle \leq 0$ for all $\left(x_{i}, y_{i}\right) \in \operatorname{gph} H, i=1,2$, that are close to $(\bar{x}, \bar{y})$. In addition assume $H$ is metrically regular at $\bar{x}$ for $\bar{y}$. Then there exists $a>0$ such that $H^{-1}(y) \cap \mathbb{B}_{a}(\bar{x})$ is a singleton for every $y \in \mathbb{B}_{a}(\bar{y})$.

Applying this result to the mapping $(t, y) \mapsto P(t, y)=S^{-1}(t, \cdot)(y) \cap \mathbb{B}_{a}(0)$, from the negative semidefiniteness of $A(t)$ we obtain that for each $t \in T$ and $y \in \mathbb{B}_{a}(0)$ the set $P(t, y)$ is a single point, hence condition a) holds. Let $y \in \mathbb{B}_{a}(0)$ and $t_{k} \rightarrow t \in T$, and let $x \in P(t, y)$. Then $y+\left(A\left(t_{k}\right)-A(t)\right) x \in A\left(t_{k}\right) x+N_{Q}(x)$ and from the Aubin property of $S^{-1}\left(t_{k}, \cdot\right)$ and the continuity of $A(\cdot)$ we obtain that there exists $x_{k} \in P\left(t_{k}, y\right)$ such that $x_{k} \rightarrow x$. Since $P$ is single-valued, it is continuous at $t$; that is, condition b) holds. Thus, we can apply Theorem 6 , obtaining that, in the current notation, we again have equality (8). For $Q=X$ the second equality becomes yet again the Bartle-Graves theorem.

\section{REFERENCES}

1. R. G. Bartle and L. M. Graves, Mappings between function spaces, Trans. Amer. Math. Soc. 72 (1952), 400-413. MR 13:951i

2. J. M. BorweIn, Stability and regular points of inequality systems, In: Fourth Symposium on nonlinear programming with data perturbations, J. of Optim. Theory and Appl. 48 (1986), 9-52. MR 87m:58018

3. J. M. Borwein, Norm duality for convex processes and applications, In: Fourth Symposium on nonlinear programming with data perturbations, J. of Optim. Theory and Appl., 48 (1986), 53-64. MR 87d:90126

4. A. L. Dontchev, The Graves theorem revisited, J. of Convex Analysis 3 (1996), 45-53. MR 97g:46055

5. A. L. Dontchev, A. S. Lewis, R. T. Rockafellar, The radius of metric regularity, Trans. Amer. Math. Soc. 355 (2003), 493-517.

6. N. Dunford, J. T. Schwartz, Linear Operators. I. General Theory. With the assistance of W. G. Bade and R. G. Bartle. Pure and Applied Mathematics, Vol. 7, Interscience Publishers, Inc., New York; Interscience Publishers, Ltd., London, 1958. MR 22:8302

7. L. M. Graves, Some mapping theorems, Duke Math. J. 17 (1950), 111-114. MR 11:729e

8. A. D. Ioffe, Metric regularity and subdifferential calculus, Uspekhi Mat. Nauk 55 (2000), no. 3 (333),103-162; English translation, Math. Surveys 55 (2000), 501-558. MR 2001j:90002

9. L. A. Lusternik, On the conditional extrema of functionals, Math. Sbornik 41 (1934), 390401 (in Russian). 
10. Z. PÁLES, Inverse and implicit function theorems for nonsmooth maps in Banach spaces, J. Math. Anal. Appl. 209 (1997), 202-220. MR 98b:49018

11. R. T. Rockafellar, R. J.-B. Wets, Variational Analysis, Springer-Verlag, Berlin, 1997. MR 98m:49001

FRsC, Canada Research Chair in Information Technology, Centre for Experimental and Constructive Mathematics, Department of Mathematics, Simon Fraser University, Burnaby, British Columbia, Canada V5A 1S6

Mathematical Reviews, Ann Arbor, Michigan 48107-8604 